PROCEEDINGS OF THE AMERICAN MATHEMATICAL SOCIETY

Volume 124, Number 8, August 1996

\title{
COMPLETELY POSITIVE PROJECTIONS ON A HILBERT SPACE
}

\author{
YASUHIDE MIURA
}

(Communicated by Palle E. T. Jorgensen)

\begin{abstract}
The purpose of this paper is to prove that a completely positive projection on a Hilbert space associated with a standard form of a von Neumann algebra induces the existence of a conditional expectation of the von Neumann algebra with respect to a normal state, and we consider the application to a standard form of an injective von Neumann algebra.
\end{abstract}

\section{INTRODUCTION}

Many authors have studied the problem of how an algebraic structure of a von Neumann algebra is determined by the underlying Hilbert space. Connes [C1] introduced an orientation of a homogeneous selfdual cone in a Hilbert space and characterized the Hilbert space associated with a standrd form of a von Neumann algebra. A geometric interpretation was given by Iochum [I1] to an algebraic notion of a conditional expectation (i.e., a projection of norm one) of a von Neumann algebra by using an orientation property in a selfdual cone.

On the other hand, Schmitt and Wittstock [SW] characterized a matrix-ordered Hilbert space with a family of selfdual cones arising from standard forms of von Neumann algebras by a projection property instead of orientation. Matrix-ordered spaces were first introduced by Choi and Effros $[\mathrm{CE}]$ as the appropriate objects to which completely positive maps apply and enabled us to handle non-commutative order. In $[\mathrm{M}]$ the author proved that a faithful normal conditional expectation with respect to a cyclic and separating vector on a von Neumann algebra induces the existence of a completely positive projection on the Hilbert space, and considered an approximation property in $L^{2}(M)$ with respect to a completely positive map when $M$ is an injective factor. In the present note we shall consider the converse theorem.

We shall use the book of Takesaki [T2] as a reference of the standard results of operator algebras. We shall use the notion as introduced in [SW] with respect to matrix-ordered standard forms and their construction.

Received by the editors October 12, 1994 and, in revised form, February 28, 1995.

1991 Mathematics Subject Classification. Primary 46L10.

Key words and phrases. Completely positive map, selfdual cone, conditional expectation, standard form of von Neumann algebra, injective von Neumann algebra.

(C)1996 American Mathematical Society 


\section{RESULTS}

Throughout this section we assume that $\left(M, H, H_{n}^{+}, n \in \mathbb{N}\right)$ is a matrix-ordered standard form [SW, Chapter 1] of a $\sigma$-finite $W^{*}$-algebra $M$. Let $e$ be a completely positive projection on $H$, i.e., $e_{n}=e \otimes 1_{n}$ maps $H_{n}^{+}$into $H_{n}^{+}$for every $n \in \mathbb{N}$ where $1_{n}$ denotes the identity on the $n \times n$ matrices. We set $K=e H, K_{n}=e_{n} H_{n}$, $K_{n}^{+}=e_{n} H_{n}^{+}, n \in \mathbb{N}$.

We need two lemmata to prove the main theorem.

Lemma 1. If $\left(K, K_{n}^{+}, n \in \mathbb{N}\right)$ is as above, then there exists a von Neumann algebra $N$ such that $\left(N, K, K_{n}^{+}, n \in \mathbb{N}\right)$ is a matrix-ordered standard form.

Proof. It is straightforward to see that $\left(K, K_{n}^{+}, n \in \mathbb{N}\right)$ is a matrix-ordered Hilbert space with selfdual cones. It suffices by [SW, Theorem 4.3] to prove that any closed face of each selfdual cone $K_{n}^{+}(n \in \mathbb{N})$ is projectable. Let $F$ be a closed face of $K_{n}^{+}$. The face generated by $F$ in $H_{n}^{+}$is denoted by $\langle F\rangle$. We then obtain by [I2, II.1.7 Lemma and II.1.3 Proposition] and [C1, Theorem 4.2] the inclusion

$$
\begin{aligned}
P_{F} K_{n}^{+} & =e_{n} P_{\langle F\rangle} e_{n} K_{n}^{+} \subset e_{n} P_{\langle F\rangle} H_{n}^{+}=e_{n} P_{F^{\perp \perp}} H_{n}^{+} \\
& =e_{n} F^{\perp \perp}=e_{n} \overline{\langle F\rangle}=\bar{F}=F,
\end{aligned}
$$

where $P_{F}$ (resp. $\left.P_{\langle F\rangle}\right)$ denotes the orthogonal projection of $K_{n}$ (resp. $H_{n}$ ) onto the closed subspace generated by $F$ (resp. $\langle F\rangle$ ).

Lemma 2. Let $M, e$ and $N$ be as above. Assume, in addition, that $e \xi_{0}=\xi_{0}$ for some cyclic and separating vector $\xi_{0}$ in $H^{+}$for $M$. If we put $L=M \cap\{e\}^{\prime}$, then $L|e H=e M| e H=N$.

Proof. It is easy to see that $J e=e J, e J \mid K=J_{K^{+}}, J_{n} e_{n}=e_{n} J_{n}$ and $e_{n} J_{n} \mid K_{n}=$ $J_{K_{n}^{+}}$.

We shall first prove that $e M \mid K \subset N$. Take a derivation $\delta$ in $D\left(H_{2}^{+}\right)$. We see from [C1, Lemma 5.3] that $e_{2} \delta e_{2} J_{2}=J_{2} e_{2} \delta e_{2}$ and if $\eta, \zeta \in K_{2}^{+}$and $(\eta, \zeta)=0$, then $\left(e_{2} \delta e_{2} \eta, \zeta\right)=(\delta \eta, \zeta)=0$. This implies that $e_{2} \delta \mid K_{2}$ belongs to $D\left(K_{2}^{+}\right)$. By virtue of the standard form $\left(M_{2}(M), H_{2}, J_{2}, H_{2}^{+}\right)$we see from [C1, Theorem $\left.3.4 \mathrm{c}\right)$ ] that for each element $X=\left[\begin{array}{ll}x & 0 \\ 0 & 0\end{array}\right]$ in $M_{2}(M)$ there exists $Y=\left[\begin{array}{ll}a & b \\ c & d\end{array}\right] \in M_{2}(N)$ satisfying $e_{2}\left(X+J_{2} X J_{2}\right) \Xi=\left(Y+J_{K_{2}^{+}} Y J_{K_{2}^{+}}\right) \Xi$ for all $\Xi \in K_{2}$. By setting $\Xi=\left[\begin{array}{ll}0 & \xi \\ 0 & 0\end{array}\right]$ with $\xi \in K$ we have

$$
\left[\begin{array}{cc}
0 & e x \xi \\
0 & 0
\end{array}\right]=\left[\begin{array}{cc}
J b J \xi & (a+J d J) \xi \\
0 & c \xi
\end{array}\right]
$$

so that $b=c=0$ and $e x \xi=a \xi+J d J \xi$. Moreover, if we set $\Xi=\left[\begin{array}{ll}0 & 0 \\ 0 & \xi\end{array}\right]$, then $\left[\begin{array}{ll}0 & 0 \\ 0 & 0\end{array}\right]=\left[\begin{array}{cc}0 & 0 \\ 0 & (d+J d J) \xi\end{array}\right]$. It follows that $e x \xi=a \xi-d \xi$ for all $\xi \in K$, whence $e M \mid K \subset N$.

We next want to prove that $N \subset L \mid K$. Take any projection $p$ in $N$. Since in general by [C1, Theorem 4.2] the map $q \rightarrow q J q J H^{+}$is an order isomorphism of the set of all projections of $M$ onto the set of all closed faces of $H^{+}$, we see that $\left[\begin{array}{ll}p & 0 \\ 0 & 1\end{array}\right] J_{K_{2}^{+}}\left[\begin{array}{ll}p & 0 \\ 0 & 1\end{array}\right] J_{K_{2}^{+}} K_{2}^{+}$is a closed face of $K_{2}^{+}$, which will be denoted by $F$, and $P_{F}=\left[\begin{array}{ll}p & 0 \\ 0 & 1\end{array}\right] J_{K_{2}^{+}}\left[\begin{array}{ll}p & 0 \\ 0 & 1\end{array}\right] J_{K_{2}^{+}}$. There then exists a projection $P=\left[\begin{array}{cc}a & b \\ b^{*} & c\end{array}\right]$ in $M_{2}(M)$ such that $P_{\langle F\rangle}=P J_{2} P J_{2}$. It follows from [I2, II.1.7 Lemma] that $P_{F} \Xi=e_{2} P_{\langle F\rangle} \Xi$ for all $\Xi \in K_{2}$. By setting $\Xi=\left[\begin{array}{ll}0 & \xi \\ 0 & 0\end{array}\right]$ we have

$$
p \xi=e a J c J \xi
$$


for all $\xi \in K$. On the other hand, since $e_{2} P_{F} e_{2} \leq P_{\langle F\rangle}$ we have for all $\xi \in K$

$$
\begin{aligned}
{\left[\begin{array}{ll}
0 & 0 \\
0 & \xi
\end{array}\right] } & =\left[\begin{array}{ll}
p & 0 \\
0 & 1
\end{array}\right] J_{K_{2}^{+}}\left[\begin{array}{ll}
p & 0 \\
0 & 1
\end{array}\right] J_{K_{2}^{+}}\left[\begin{array}{ll}
0 & 0 \\
0 & \xi
\end{array}\right] \\
& =\left[\begin{array}{cc}
a & b \\
b^{*} & c
\end{array}\right] J_{2}\left[\begin{array}{cc}
a & b \\
b^{*} & c
\end{array}\right] J_{2}\left[\begin{array}{ll}
0 & 0 \\
0 & \xi
\end{array}\right]=\left[\begin{array}{lll}
b J b J \xi & b J c J \xi \\
c J b J \xi & c J c J \xi
\end{array}\right] .
\end{aligned}
$$

We then have by [SW, Corollary 3.3] $b \xi=0$. Since $K^{+}$contains by assumption a cyclic and separating vector for $M$, we have $b=0$. It follows that both $a$ and $c$ must be projections because $P$ is a projection. Since

$$
\xi=c J c J \xi=c \xi
$$

for all $\xi \in K$, we have by assumption $c=1$. Hence the equality $(*)$ implies $p \xi=e a \xi$ for all $\xi \in K$. We see from [I2, II.1.7 Lemma] that $e_{2} P_{\langle F\rangle}=P_{\langle F\rangle} e_{2}$, i.e. $\left[\begin{array}{cc}e a J a J & e a \\ e J a J & e\end{array}\right]=\left[\begin{array}{ccc}a J a J e & a e \\ J a J e & e\end{array}\right]$, whence $e a=a e$. Therefore, for every element $x$ in $N$ there exists an element $y$ in $M$ commuting with $e$ such that $x \xi=y \xi$ for all $\xi \in K$.

Consequently, the argument above shows that

$$
L|K \subset e M| K \subset N \subset L \mid K .
$$

Theorem 3. Let $\left(M, H, H_{n}^{+}, n \in \mathbb{N}\right)$ be a matrix-ordered standard form of the von Neumann algebra $M$, and let e be a completely positive projection on $H$ satisfying $e \xi_{0}=\xi_{0}$ for some cyclic and separating vector $\xi_{0} \in H^{+}$for $M$. If $L=M \cap\{e\}^{\prime}$, then $\left(L \mid e H, e H, e_{n} H_{n}^{+}, n \in \mathbb{N}\right)$ is a matrix-ordered standard form. In addition, there exists a faithful normal conditional expectation $\varepsilon$ of $M$ onto $L$ such that $\omega_{\xi_{0}} \circ \varepsilon=\omega_{\xi_{0}}$. Furthermore, we have $L|e H=e M| e H$.

Proof. This part of the proof is due to Iochum [I1, Theorem 3.1.6]. For any element $x$ in $M$ there exists by Lemma $2 \alpha(x)$ in $L$ such that $e x \xi=\alpha(x) \xi$ for all $\xi$ in $K$. $\alpha(x)$ is uniquely determined since $e \xi_{0}=\xi_{0}$ is a separating vector for $M$. We then have for all $y$ in $L$

$$
\begin{aligned}
\left(e x^{*} \xi_{0}, J y J \xi_{0}\right) & =\left(\xi_{0}, x J y J \xi_{0}\right)=\left(\xi_{0}, e x J y J \xi_{0}\right) \\
& =\left(\alpha(x)^{*} \xi_{0}, J y J \xi_{0}\right) .
\end{aligned}
$$

Hence $e x^{*} \xi_{0}=\alpha(x)^{*} \xi_{0}$ because of the density of $J L J \xi_{0}$ in $K$. It follows that

$$
e S x \xi_{0}=e x^{*} \xi_{0}=\alpha(x)^{*} \xi_{0}=S \alpha(x) \xi_{0}=S e x \xi_{0} .
$$

Since $M \xi_{0}$ is a core set of $S$, we have $e S=S e$. Hence $\Delta_{\xi_{0}} e=e \Delta_{\xi_{0}}$ and $L \xi_{0}$ is invariant under $\Delta_{\xi_{0}}^{i t}(\forall t \in \mathbb{R})$. From the theorem of Takesaki [T1, Theorem] we see the existence of the conditional expectation $\varepsilon$. This completes the proof.

Note that in general $e$ does not belong to $M$. As an application of the above theorem we immediately obtain the following corollary in which we consider a von Neumann algebra from the point of view of semidiscreteness in the related $L^{2}$-space.

Corollary 4. Let $\left(M, H, H_{n}^{+}, n \in \mathbb{N}\right)$ be a matrix-ordered standard form of the von Neumann algebra $M$. If there exists an increasing sequence $\left\{e^{(n)}\right\}$ of completely positive projections of finite rank on $H$ which converges strongly to 1 such that $e^{(1)} \xi_{0}=\xi_{0}$ for some cyclic and separating vector $\xi_{0} \in H^{+}$for $M$, then $M$ is injective. 
Proof. By assumption we have for all $n \in \mathbb{N} e^{(n)} \xi_{0}=e^{(n)} e^{(1)} \xi_{0}=e^{(1)} \xi_{0}=\xi_{0}$. It follows from Theorem 3 that for each $n$ there exists a finite-dimensional von Neumann subalgebra $L_{n}$ of $M$ satisfying $L_{n}\left|e^{(n)} H=e^{(n)} M\right| e^{(n)} H$. Since $L_{n} \xi_{0}=$ $e^{(n)} M \xi_{0}$ and $\xi_{0}$ is a separating vector for $M$, we have $L_{n} \subset L_{n+1}$. Thus we have $M=\left\{\bigcup_{n} L_{n}\right\}^{-s}$. So $M$ is injective.

We have many results for injectivity in the theory of operator algebras (cf. for example, $[\mathrm{CE}],[\mathrm{C} 2])$. In [S] Schmitt studied the Arveson space, which is a Banach space having the completely positive extension property with predual being a certain matrix-ordered Banach space, via several equivalent properties which are the finite injectivity, the approximative factorization property, the matricial Riesz interpolation property and the matricial Hahn-Banach property, and gave the characterization of matrix-ordered standard forms of injective $W^{*}$-algebras.

\section{ACKNOWLEDGMENT}

The author wishes to express his sincere gratitude to Professor B. Iochum for having pointed out this problem to him. He thanks the members of the seminar in Sendai, especially Professors T. Okayasu, K. Saito, and H. Takemoto, for their useful suggestions. Thanks are also due to the referee for valuable comments.

\section{REFERENCES}

[A] H. Araki, Some properties of modular conjugation operator of von Neumann algebras and a non-commutative Radon-Nikodym theorem with a chain rule, Pacific J. Math. 50 (1974), 309-354. MR 50:2929

[CE] M. D. Choi and E. G. Effros, Injectivity and operator spaces, J. Funct. Anal. 24 (1977), 156-209. MR 55:3814

[C1] A. Connes, Caractérisation des espaces vectoriels ordonnées sous-jacents aux algèbres de von Neumann, Ann. Inst. Fourier 24 (1974), 121-155. MR 51:13705

[C2] Classification of injective factors. Cases $\mathrm{II}_{1}, \mathrm{II}_{\infty}, \mathrm{III}_{\lambda}, \lambda \neq 1$, Ann. of Math. (2) 104 (1976), 73-115. MR 56:12908

[H] U. Haagerup, The standard form of von Neumann algebras, Math. Scand. 37 (1975), 271283. MR 53:11387

[I1] B. Iochum, Cônes autopolaires dans les espaces de Hilbert, Thèse, Univ. de Provence Centre de Saint-Charles, 1975.

[I2] C Cônes autopolaires et algèbres de Jordan, Lecture Notes in Math., vol. 1049, Springer-Verlag, Berlin, Heidelberg, New York, and Tokyo, (1984). MR 86m:46067

[M] Y. Miura, A certain factorization of selfdual cones associated with standard forms of injective factors, Tokyo J. Math. 13 (1990), 73-86. MR 92d:46161

[MT] Y. Miura and J. Tomiyama, On a characterization of the tensor product of the selfdual cones associated to the standard von Neumann algebras, Sci. Rep. Niigata Univ. Ser. A 20 (1984), 1-11. MR 85j:46102

[S] L. M. Schmitt, Characterization of $L^{2}(\mathcal{M})$ for injective $W^{*}$-algebras $\mathcal{M}$, Math. Scand. 57 (1985), 267-280. MR 87m:46116

[SW] L. M. Schmitt and G. Wittstock, Characterization of matrix-ordered standard forms of $W^{*}$-algebras, Math. Scand. 51 (1982), 241-260. MR 84i:46062

[T1] M. Takesaki, Conditional expectations in von Neumann algebras, J. Funct. Anal. 9 (1972), 306-321. MR 46:2445

[T2] Theory of operator algebras, I, Springer-Verlag, New York, Heidelberg, and Berlin, (1979). MR 81e:46038

Department of Mathematics, College of Humanities and Social Sciences, Inate UniVERSity, MORIOKA 020, JAPAN 